\title{
Radar Target Characteristics Extraction using Polarization Scattering Matrix
}

\author{
L. Vijaya Lakshmi ${ }^{*}$ A. Jaya Lakshmi, M. N. V. S. S. Kumar, P. Sirish Kumar \\ Department of Electronics and Communication Engineering, \\ Aditya Institute of Technology and Management (AITAM), Tekkali, India \\ *Corresponding author, e-mail: lingala.vijju@gmail.com
}

\begin{abstract}
Now a day's characterization of targets using radar is very important in Air Traffic Control, Defense, and Stealth etc. In order to know the characteristics of the target it is very essential to know the polarization properties of that particular target which depends upon scattering nature of the target. The polarization properties are important for radar target besides amplitude, phase and frequency. The polarization may be potentially used to improve target detection, anti-interference, and radar target recognition. Polarization properties of a target can be obtained using polarization scattering matrix (PSM). In this paper the polarization matrix of various geometrical shapes are derived. For radar target recognition (RTR), a method using properties of the polarization scattering matrix (PPSM) is presented in this paper. $A$ dipole has been considered to calculate the polarization matrix and polarization properties. The properties of the polarization scattering matrix: the determinant, Trace of Power Scattering Matrix, Depolation, Eigen polarization angle and Module of Polarization Ellipticity are analyzed. These properties are analyzed for different orientation angles of the targets.
\end{abstract}

Keywords: scattering matrix, polarization

Copyright $@ 2015$ Institute of Advanced Engineering and Science. All rights reserved.

\section{Introduction}

The word RADAR is an acronym from the words RAdio Detection and Ranging, i.e. finding and positioning a target and determining the distance (range) between the target and the radar by using radio frequency. The basic principle behind the radar is simple: transmitter sends out a very short duration pulse at a high power level. The pulse strikes an object (or a target) and energy will be reflected (known as radar returns or echoes) back to the radar receiver. These echoes are then processed by the radar receiver to extract target information such as range, velocity, angular position, and other target identifying characteristics. Radar Cross Section is used to measure the characteristics of the targets. The response of the radar target is profoundly influenced by the operating frequency, the target orientation relative to the radar system, and the radar waveform and processing. RCS is a scalar number which is a function of Polarization of incident and received waves. The interaction of the incident wave and target is given by polarization scattering matrix. Both linear and circular polarizations are of interest, and also, a combination of the two, wherein circular polarization is transmitted, while a two-channel receiver measures orthogonal linear polarization components. The measurement of the polarization is called polarimetry. Radar Polarimetry [4] is the science of acquiring, processing and analyzing the polarization state of an electromagnetic field. In-depth study of radar cross section in terms of transmitted and received polarizations in an attempt to better understand the potential of polarimetric radar. The polarization scattering matrix, is a generalization of the concept of radar cross section, and includes amplitude, phase, and polarization. In this paper the polarization scattering matrices are derived for the simple target: the dipole. It is known that polarization properties depend on the object's shape, size, composition and the radar characteristics. For a given set of radar characteristics, the scattering matrix of a target permits prediction of radar return for any polarization. In this paper the polarization matrix for dipole is derived. For characterizing the RTR, a method using properties of the polarization scattering matrix (PPSM) is presented in this paper. The properties of the polarization scattering matrix: the determinant, Trace of Power Scattering Matrix, Depolation, Eigen polarization angle and 
Module of Polarization Ellipticity are analyzed. These properties are analyzed for different orientation angles of the targets.

\section{Determination of Target Properties}

Radar target recognition (RTR) is the needs of new radar [2-3]. While RTR is also one of the most difficult problems for radar, so it is very necessary to explore new algorithm for RTR. Radar Polarimetry (Polar: polarization, Metry: measure) is the science of acquiring, processing and analyzing the polarization state of an electromagnetic field. The polarization properties are important for radar target besides amplitude, phase and frequency. Polarimetry deals with the vectorial nature of electromagnetic field, so some problems cannot be solved in time domain, frequency domain may be solve in polarization domain. The polarization may be potentially used to improve target detection, anti-interference, and RTR.

It is known that polarization properties depend on the object's shape, size, and composition, and upon the radar characteristics. The full polarization radar can receive all the electromagnetic backward wave of targets, while the mono-polarization can not. For a given set of radar characteristics, the scattering matrix of a target permits prediction of radar return for any polarization. One example for RTR is that the ball which can be the same RCS as a cone object is not identified while the polarization radar can be easily discovered, because the scattering matrix of ball at any azimuth angles and pitch angles is the same, while the scattering matrix of a cone target varied with azimuth angles and pitch angles.

In this project scattering matrix of various geometrical shapes are obtained and then PPSMs are analyzed.

\subsection{Polarization Scattering Matrices for Simple Target}

In this section, we derive monostatic scattering matrices for a dipole and a dihedral corner reflector. Results are presented for both linear and circular polarizations. It is noted that these scattering matrices provide for partitioning polarization and for the phase change of each polarization component, but they do not account for amplitude. To account for amplitude, the scattering matrix must be multipled by a real non-negative constant. This constant can be estimated in the following manner: First determine the cross section of the target under consideration, via the procedures given in many radar texts- (this cross section is usually given for same sense linearly polarized transmitted and received signals), and then scaled via the radar range equation; the constant is the square root of this scaled cross section.

a) Dipole target

Assume that a thin wire (diameter $\ll<$ wavelength) or a sharp edge of a conducting body is constrained to lie parallel to the $\hat{x}, \hat{y}$ plane and at an angle $\gamma$ with respect to the $\hat{x}, \hat{z}$ plane (see Figure 1). Assume further that a transmitted field $\bar{E}^{T}=H^{T} \hat{x}+V^{T} \hat{y}$ is normally incident on the wire. For such a field, the induced current along the dipole is proportional to the projection of incident electric field along the axis of the dipole. The reradiated field is proportional to the induced current, and it is polarized along the axis of the dipole. Thus, the induced current, $i_{d}$, is expressed as:

$$
i_{d} \propto H^{T} \cos \gamma+V^{T} \sin \gamma
$$

And the reradiated field, $\bar{E}_{d}$ is given by:

$$
\bar{E}_{d} \propto\left(H^{T} \cos \gamma+V^{T} \sin \gamma\right) \hat{\gamma}
$$

Where $\hat{\gamma}$ is a unit vector parallel to the wire. This, unit vector, expressed in terms of $\hat{x}$ and $\hat{y}$, can be written:

$$
\hat{\gamma} \propto \cos \gamma \hat{x}+\sin \gamma \hat{y}
$$


The reradiated field and the received field $\bar{E}^{R}=H^{R} \hat{x}+V^{R} \hat{y}$, are also proportional; therefore,

$$
\begin{aligned}
& H^{R} \propto H^{T} \cos ^{2} \gamma+V^{T} \sin \gamma \cos \gamma \\
& V^{R} \propto H^{T} \sin \gamma \cos \gamma+V^{T} \sin ^{2} \gamma
\end{aligned}
$$

The dipole scattering matrix, $\left[\mathrm{S}_{\llcorner}\right]$dipole for linear representation of polarization is given by:

$$
\left[S_{L}\right]_{\text {dipole }}=\left[\begin{array}{cc}
\cos ^{2} \gamma & \sin \gamma \cos \gamma \\
\sin \gamma \cos \gamma & \sin ^{2} \gamma
\end{array}\right]
$$

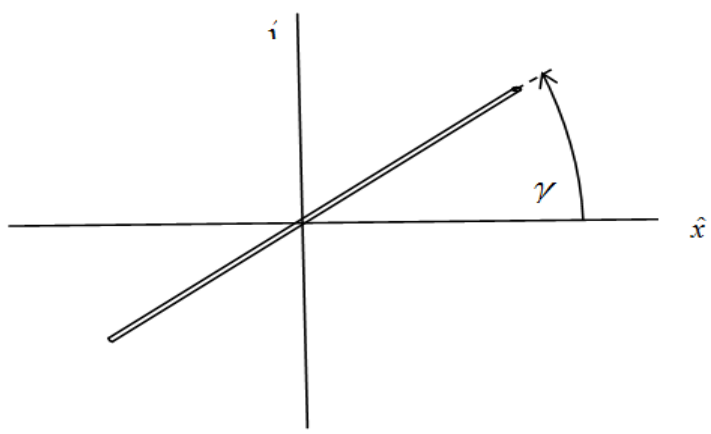

Figure 1. Dipole Parallel to $\hat{x}$ and $\hat{y}$ given by:

The dipole scattering matrix, $\left[\mathrm{S}_{\mathrm{c}}\right]$ dipole, for circular representation of polarization is

$$
\left[S_{C}\right]_{\text {dipole }}=\left[\begin{array}{cc}
e^{-j 2 \gamma} & 1 \\
1 & e^{j 2 \gamma}
\end{array}\right]
$$

\section{Properties of the Polarization Scattering Matrix}

In the $H-V$ basis [1], the relationship between the incident and reflected fields may be expressed as:

$$
E_{S}=[S] E_{i}
$$

Where $E_{s}$ is the reflected field, $E_{i}$ is the incident field, the fields are related by the Sinclair matrix $[S]$

$$
S=\left[\begin{array}{ll}
S_{H H} & S_{H V} \\
S_{V H} & S_{V V}
\end{array}\right]
$$

For mutually object: $S_{H V} \approx S_{V H}$

Then the IPPSMs are obtained

1) Determinant $|\Delta|$

$$
|\Delta|=|\operatorname{det}[S]=| S_{H H} S_{V V}-S^{2}{ }_{H V} \mid
$$

2) Trace of Power Scattering Matrix $P_{1}$ 
$P_{1}=\left|S_{H H}\right|^{2}+\left|S_{v v}\right|^{2}+2\left|S_{H V}\right|^{2}$

3) Depolation $D$

$D=1-\frac{\left|S_{H H}+S_{V V}\right|^{2}}{2 P_{1}}$

4) Eigen- polarization angle

$\varphi_{0}=\frac{1}{2} \arctan \frac{2 \operatorname{Re}\left(S_{1} * S_{12}\right)}{\operatorname{Re}\left(S_{1} * S_{2}\right)}$

where.* denotescomplexconjugatia,

$\left\{\begin{array}{l}S_{1}=S_{H H}+S_{V V} \\ S_{2}=S_{H H}-S_{V V}\end{array}\right.$

5) Eigen- polaization Ellipticity $\tau_{0}$

$\tau_{0}=\frac{1}{2} \arctan \frac{j 2 S_{12}^{*}}{S_{1}}$

Where,

$S_{12}^{*}=S_{12} \cos \left(2 \varphi_{0}\right)-\frac{1}{2} S_{2} \sin \left(2 \varphi_{0}\right)$

All the physical characteristics of PPSMS is given in Table 1.

Table 1. Physical characteristic of IPPSMs

\begin{tabular}{ll}
\hline PPSMs & Physical characters \\
\hline$\Delta$ & It means 'fat' or 'thin' of target \\
$P_{1}$ & It means 'small' or 'big' of target \\
$D$ & $\begin{array}{l}\text { It means the number of scattering centers, while } \\
0<D<0.5 \text { as to one scattering center, } 0.5<D<1 \text { as to } \\
\text { two or more scattering centers }\end{array}$ \\
$\varphi_{0}$ & $\begin{array}{l}\text { It measures the relative orientation between the } \\
\text { antenna and the }\end{array}$ \\
& axis of the eigen-polarization ellipse \\
$\tau_{0}$ & It relates to the symmetry of the target \\
\hline
\end{tabular}

\section{Result}

\subsection{Dipole Polarization Properties for linear polarization}

Figure 2 shows the full polarization amplitude of a dipole. From this figure we can see that amplitude difference of $45 \mathrm{~dB}$ for co-polarization and amplitudes of cross polarizations are equal.

The determinant and trace of power are shown in Figure 3 and Figura 4. The determinant and trace of power scattering matrix $\mathrm{P}_{1}$ are invariant with orientation varied in $0^{0}$ to $30^{\circ}$. 


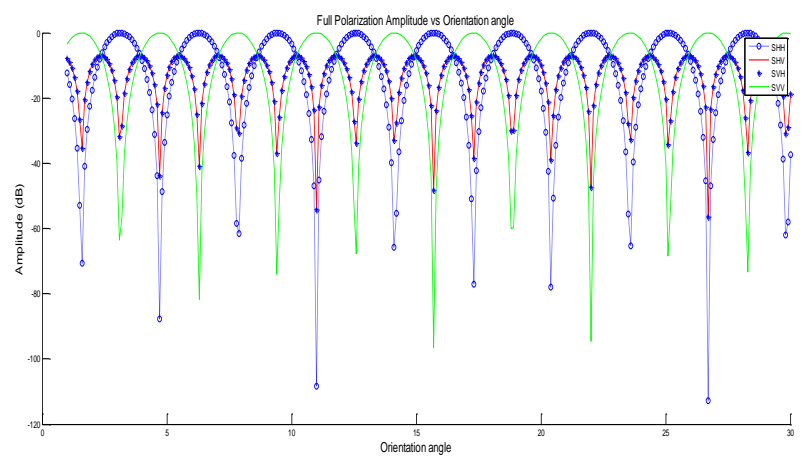

Figure 2. Full Polarization Amplitudes of a Dipole

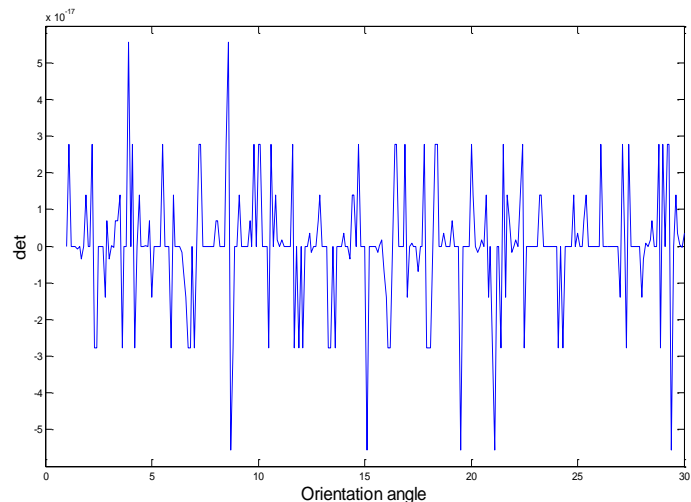

Figure 3. Variation of determinant with orientation angle

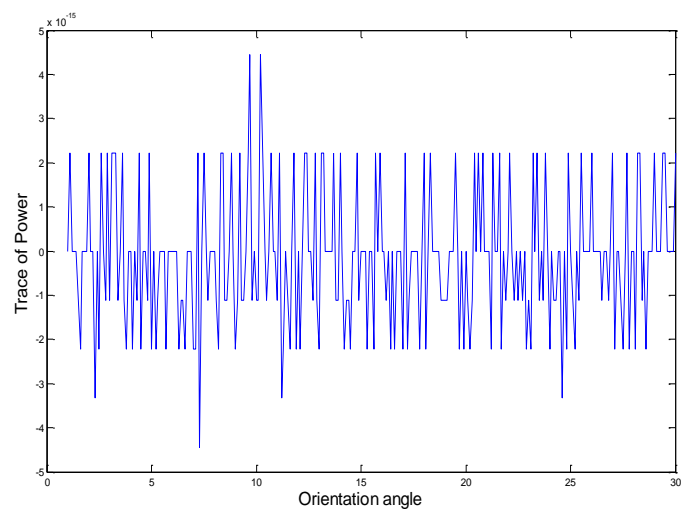

Figure 4. Variation of Trace Power $P_{1}$ with Orientation Angle

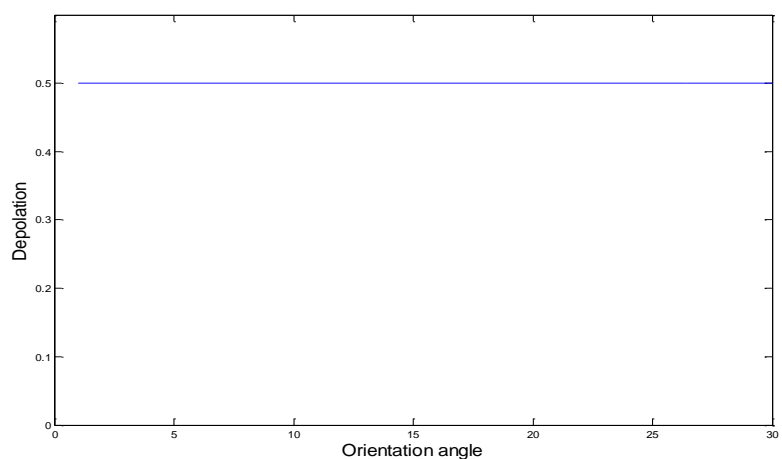

Figure 5.Depolation vs Orientation angle

From above figure the value of depolation is 0.5 . With this one can say that the scattering centre for a dipole is one.

The eigen polarization angle $\varphi_{0}$ in radians and eigen polarization elipticity are shown in is Figures 6 and Figure 7. The eigen polarization angle varies from $-44^{\circ}$ to $44^{\circ}$. The eigen polarization ellipticity varies from -2 to 2 symmetrically and regularly for dipole. 


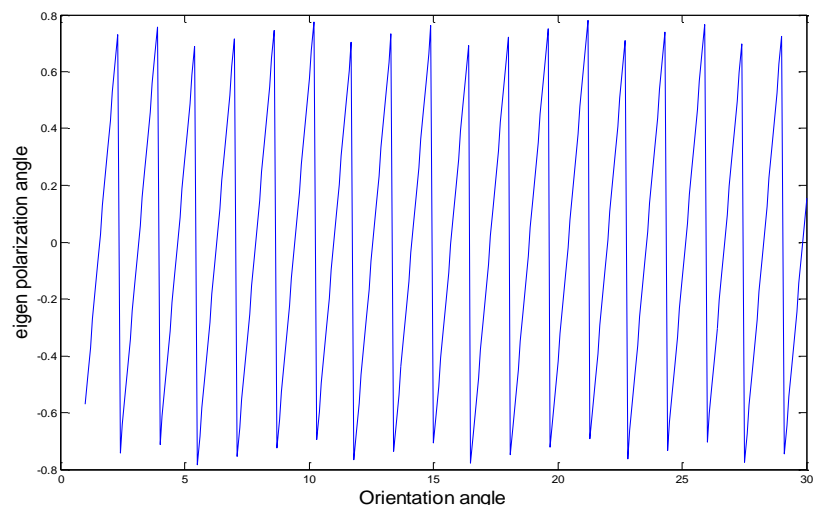

Figure 6. Variation of Eigen Polarization Angle with Orientation Angle

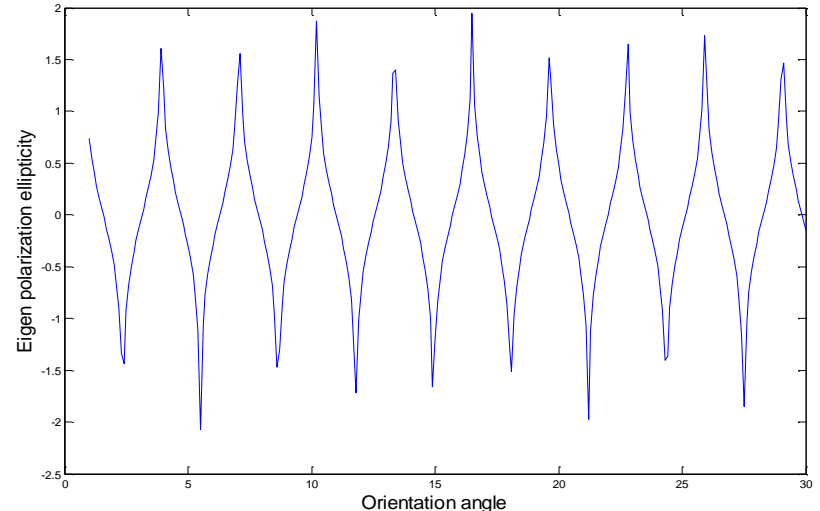

Figure 7. Variation of Eigen Polarization Ellipticity with Orientation Angle

\subsection{Dipole Polarization Properties for Circular Polarization}

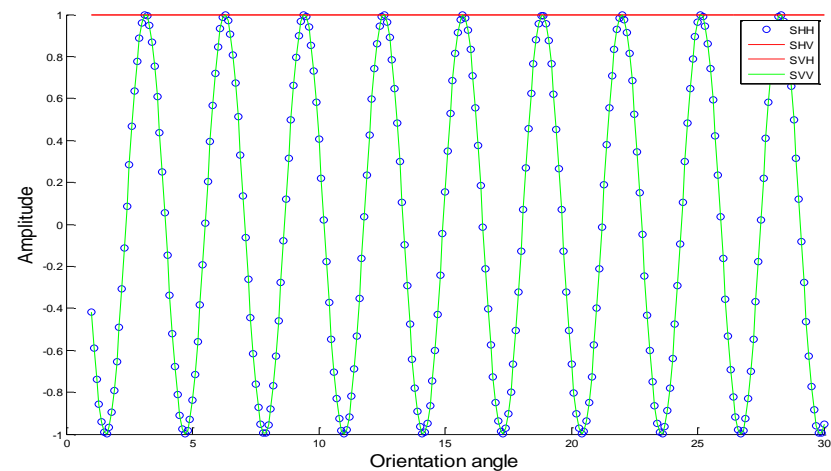

Figure 8. Full Polarization Amplitudes of a Dipole 


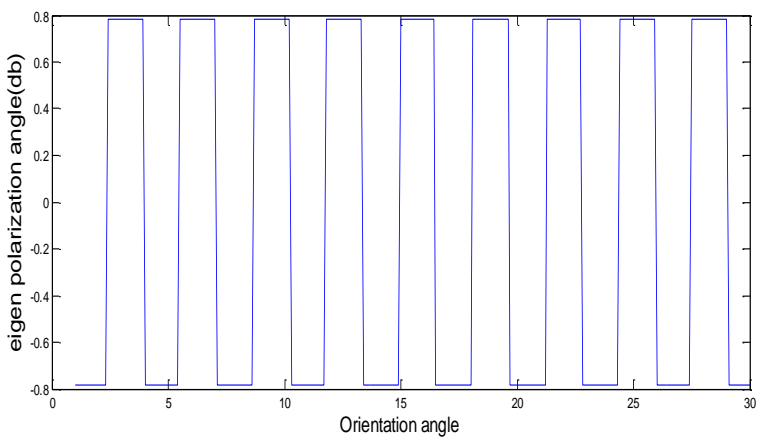

Figure 9. Variation of Determinant with Orientation Angle

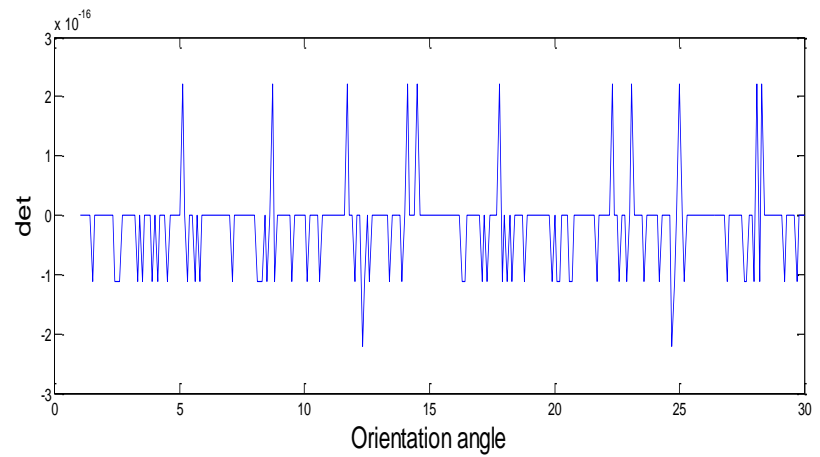

Figure 10. Variation of Trace Power $P_{1}$ with Orientation Angle

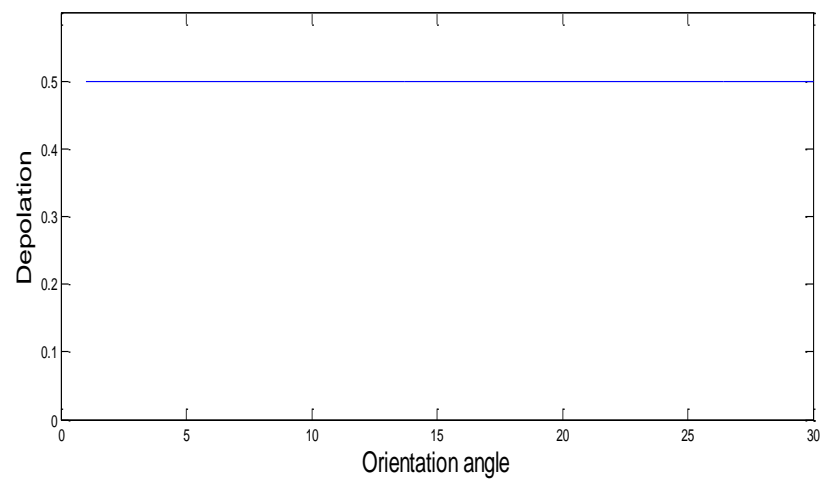

Figure 11. Depolation vs Orientation Angle

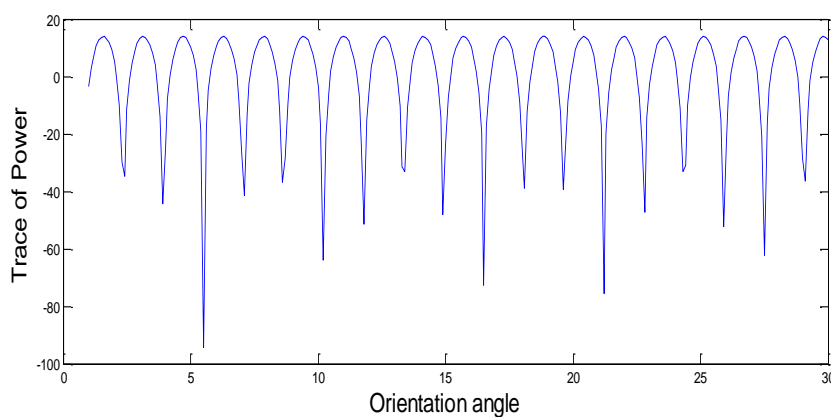

Figure 12. Variation of Eigen Polarization Angle with Orientation Angle

TELKOMNIKA Vol. 16, No. 2, November 2015 : $308-315$ 


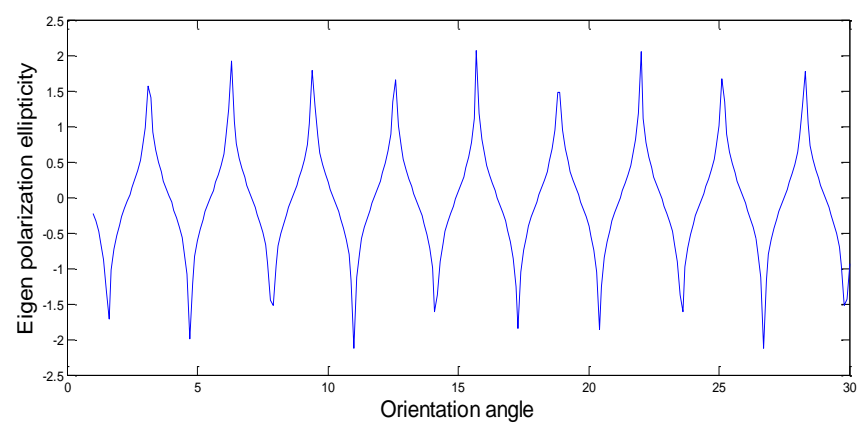

Figure 13. Variation of Eigen Polarization Ellipticity with Orientation Angle

Figure 8 to 13 shows the properties of polarization matrix for circular polarization of the dipole.

\section{Conclusion}

In this paper, polarization properties of a dipole are obtained using polarization scattering matrix (PSM). This helps in indentifying the different objects having same RCS. Polarization properties are determined for both linear and circular polarization of dipole. The properties of the polarization scattering matrix: the determinant, Trace of Power Scattering Matrix, Depolation, Eigen polarization angle and Module of Polarization Ellipticity are determined in this paper. It is observed that the amplitudes of cross-polarization are equal and the amplitude difference of co-polarization is about $45 \mathrm{~dB}$. The determinant and trace of power scattering matrix $\mathrm{P}_{1}$ are invariant with orientation varied in $0^{\circ}$ to $30^{\circ}$. The value of depolation observed is 0.5 which indicates that the scattering centre for a dipole is one. The eigen polarization angle varies from $-44^{0}$ to $44^{\circ}$ and the eigen polarization ellipticity varies from -2 to 2 symmetrically and regularly for dipole. This indicates that the dipole is cylindrically symmetrical. The dipole polarization properties are determined assuming the radar is operating at $\mathrm{S}$ band.

\section{References}

[1] Fuyou Wang, Rujiang Guo, Yinhe Huang. Radar Target Recognition Based on Some Invariant Properties of the Polarization Scattering Matrix. IEEE. 2011.

[2] Alaee M. Amindavar H. Chirplet-based target recognition using radar technology. 2008 5th IEEE Sensor Array and Multichannel Signal Processing Workshop. 2008: 451-454.

[3] Joon-ho L, In-sik C, Hyo-tae K. Natural frequency-based neural network approach to radar target recognition signal processing. IEEE Transactions on Signal Processing. 2003; 51(12): 3191-3197.

[4] Dr George A Emmons, Dr P Martin Alexander. Polarization Scattering Matrices for Polarimetric Radar. US Army Missile Command. Alabama. 1983

[5] Bickel SH. Some Invariant Properties of the Polarization Scattering Matrix. Proc. IEEE. 1965; 53(8): 1070-1072. 